\title{
Video Article \\ Electrophysiological Assessment of Murine Atria with High-Resolution Optical Mapping
}

\author{
Kensuke Ihara ${ }^{* 1}$, Koji Sugiyama* ${ }^{* 1}$, Kentaro Takahashi ${ }^{1}$, Masahiro Yamazoe ${ }^{1}$, Tetsuo Sasano ${ }^{2}$, Tetsushi Furukawa ${ }^{1}$ \\ ${ }^{1}$ Department of Bio-informational Pharmacology, Medical Research Institute, Tokyo Medical and Dental University \\ ${ }^{2}$ Department of Biofunctional Informatics, Tokyo Medical and Dental University \\ *These authors contributed equally
}

Correspondence to: Tetsuo Sasano at sasano.bi@tmd.ac.jp

URL: https://www.jove.com/video/56478

DOI: doi:10.3791/56478

Keywords: Medicine, Issue 132, Optical mapping, Mouse, Atrium, Electrophysiological study, Atrial fibrillation, Voltage-sensitive dye, $\mathrm{Ca}^{2+}$ indicator, Action potential, Arrhythmia

Date Published: 2/22/2018

Citation: Ihara, K., Sugiyama, K., Takahashi, K., Yamazoe, M., Sasano, T., Furukawa, T. Electrophysiological Assessment of Murine Atria with HighResolution Optical Mapping. J. Vis. Exp. (132), e56478, doi:10.3791/56478 (2018).

\section{Abstract}

Recent genome-wide association studies targeting atrial fibrillation (AF) have indicated a strong association between the genotype and electrophysiological phenotype in the atria. That encourages us to utilize a genetically-engineered mouse model to elucidate the mechanism of AF. However, it is difficult to evaluate the electrophysiological properties in murine atria due to their small size. This protocol describes the electrophysiological evaluation of atria using an optical mapping system with a high temporal and spatial resolution in Langendorff perfused murine hearts. The optical mapping system is assembled with dual high-speed complementary metal oxide semiconductor cameras and high magnification objective lenses, to detect the fluorescence of a voltage-sensitive dye and $\mathrm{Ca}^{2+}$ indicator. To focus on the assessment of murine atria, optical mapping is performed with an area of $2 \mathrm{~mm} \times 2 \mathrm{~mm}$ or $10 \mathrm{~mm} \times 10 \mathrm{~mm}$, with a $100 \times 100$ resolution $(20 \mu \mathrm{m} / \mathrm{pixel}$ or $100 \mu \mathrm{m} / \mathrm{pixel})$ and sampling rate of up to $10 \mathrm{kHz}(0.1 \mathrm{~ms})$ at maximum. A 1-French size quadripolar electrode pacing catheter is placed into the right atrium through the superior vena cava avoiding any mechanical damage to the atrium, and pacing stimulation is delivered through the catheter. An electrophysiological study is performed with programmed stimulation including constant pacing, burst pacing, and up to triple extrastimuli pacing. Under a spontaneous or pacing rhythm, the optical mapping recorded the action potential duration, activation map, conduction velocity, and Ca ${ }^{2+}$ transient individually in the right and left atria. In addition, the programmed stimulation also determines the inducibility of atrial tachyarrhythmias. Precise activation mapping is performed to identify the propagation of the excitation in the atrium during an induced atrial tachyarrhythmia. Optical mapping with a specialized setting enables a thorough electrophysiological evaluation of the atrium in murine pathological models.

\section{Video Link}

The video component of this article can be found at https://www.jove.com/video/56478/

\section{Introduction}

The heart consists of 4 chambers in mammals. The upper two chambers are atria, and the lower ones are ventricles. Ventricles work as a pump to eject blood to the systemic or pulmonary circulation. Atria receive blood returning from the systemic or pulmonary veins, and assist in transporting blood into the ventricles to obtain an efficient cardiac pump function. From an electrophysiological aspect, the important function of the atria is to regulate the heart rhythm. The electrical signals originate from the sinus node located at the junction between the superior vena cava (SVC) and right atrium (RA), then propagate to the RA and the left atrium (LA), and conduct to the ventricle through the atrioventricular node and His-Purkinje conduction system.

Arrhythmias, which are heart rhythm disorders, are classified into atrial and ventricular according to their origin. Atrial fibrillation (AF) is the most common sustained form of an arrhythmia, characterized by a random and rapid excitation of the atria. Recent genetic analyses and genomewide association studies (GWAS) have shown the association between AF and genetic mutations or monopolymorphisms ${ }^{1,2,3,4}$. These findings indicate AF is at least partly associated with a genetic cause. Therefore, it is critical to evaluate the genotype-phenotype interactions in the atria using a genetically-engineered animal model. It is widely accepted that the mouse is the most established mammal for genetic modification.

The optical mapping technique has been developed to evaluate the excitation of the heart tissue. However, the observation of the murine atrium by optical mapping is hampered by its relatively small size. We attempt to achieve a detailed assessment of the murine atrium with a high temporal and spatial resolution. 


\section{Protocol}

This animal experiment was approved and performed under the regulation of the Institutional Animal Care and Use Committee of Tokyo Medical and Dental University.

\section{Preparation}

1. Stock solutions

1. Dissolve the voltage-sensitive dyes (di-4-ANEPPS, and RH237) and $\mathrm{Ca}^{2+}$ indicator (Rhod-2 AM) with $100 \%$ dimethyl sulfoxide (DMSO) to make stock solutions with concentrations of $6 \mathrm{mM}, 10 \mathrm{mM}$, and $10 \mathrm{mM}$, respectively.

2. Dissolve the excitation-contraction (E-C) uncoupler, blebbistatin, with $90 \%$ DMSO to make a $50 \mathrm{mM}$ stock solution.

3. Aliquot the stock solutions in a $0.2 \mathrm{~mL}$ PCR tube in a dark room, and replace the air in the stock tube using nitrogen gas to avoid oxidation.

4. Wrap the stock tubes in aluminum foil individually for protection from the light, and store them at $-20^{\circ} \mathrm{C}$.

2. Working solutions

1. Prepare $1 \mathrm{~L}$ of phosphate buffered saline (PBS) without $\mathrm{Ca}^{2+}\left(137 \mathrm{mM} \mathrm{NaCl}, 2.7 \mathrm{mM} \mathrm{KCl}, 8.0 \mathrm{mM} \mathrm{Na}_{2} \mathrm{HPO}_{4}\right.$, and $1.5 \mathrm{mM} \mathrm{KH}_{2} \mathrm{PO}_{4}, \mathrm{pH}^{2}$ 7.40 adjusted by $\mathrm{NaOH}$ )

2. Prepare $1 \mathrm{~L}$ of Tyrode's solution (135 mM NaCl, $5.4 \mathrm{mM} \mathrm{KCl}, 1.8 \mathrm{mM} \mathrm{CaCl}_{2}, 0.53 \mathrm{mM} \mathrm{MgCl}_{2}, 0.33 \mathrm{mM} \mathrm{NaH}_{2} \mathrm{PO}$, $5.5 \mathrm{mM} \mathrm{D}-\mathrm{glucose}$, and $5.0 \mathrm{mM}$ HEPES, pH 7.40 adjusted by $\mathrm{NaOH}$ ).

3. Filter Tyrode's solution with a $0.22 \mu \mathrm{m}$ bottle top filter and aerate it well by bubble aeration using an air stone connected to an $\mathrm{O}_{2}$ gas cylinder.

\section{Optical mapping in Langendorff-perfused hearts}

1. Assemble the optical mapping system using two complementary metal oxide semiconductor (CMOS) cameras (Figure 1a\&b)

1. Assemble the CMOS cameras, beam splitter, objective lenses, lens revolver, light source, dichroic mirrors, filters, and other parts into the optical mapping system as shown in Figure 1a\&b.

2. Select the magnification by changing the objective lens in a turret, equipped with different magnifications $(1.6 \mathrm{X}$ and $5 \mathrm{X})$. NOTE: The size of the CMOS sensor is $10 \mathrm{~mm} \times 10 \mathrm{~mm}$, with a $100 \times 100$ resolution, which means, with $5 \mathrm{X}$ objective lens, this system provides a $20 \mu \mathrm{m}$ spatial resolution.

3. Set the excitation light using a light-emitting diode (LED) lamp with a center wavelength of $530 \mathrm{~nm}$, passed through a band-pass filter $(520 / 35 \mathrm{~nm})$, and reflected with a dichroic mirror $(560 \mathrm{~nm})$

4. Record the emission signal divided by a splitter $(665 \mathrm{~nm})$, in which setting camera 1 with a long pass filter $(697 / 75 \mathrm{~nm})$ detects the fluorescence using a membrane voltage-sensitive dye (di 4-ANEPPS or RH237), and camera 2 with a band-pass filter (572/28 $\mathrm{nm}$ ) detects the signal of the $\mathrm{Ca}^{2+}$ indicator (Rhod2-AM).

2. Assemble the Langendorff perfusion circuit

1. Attach polyvinyl chloride (PVC) tubes to a peristaltic pump.

2. Put the intake side of the PVC tube into Tyrode's solution aerated with $100 \% \mathrm{O}_{2}$ as mentioned in step 1.2.3.

3. Connect the discharge side of the PVC tube to a hand-made air trap, then arrange the $5 \mu \mathrm{m}$ filter, three-way stopcocks, pressure transducer, heating glass coil, and 21-gauge blunted needle (the needle size is changeable in accordance with the aorta size) sequentially, using other PVC tubes.

4. Fill the perfusion circuit with Tyrode's solution avoiding any air bubbles in the circuit, and stop the flow until the heart is connected to the circuit.

3. Heparinization and anesthesia

1. Inject unfractionated heparin (200 IU, regardless of the body weight) intraperitoneally into a mouse using a 25 -gauge needle and 1 -mL syringe.

2. Anesthetize the mouse by an intraperitoneal injection of pentobarbital $(65 \mathrm{mg} / \mathrm{kg}) 10 \mathrm{~min}$ after the heparin injection.

4. Cannulation and perfusion

1. Place the mouse in the supine position. Confirm animals is anesthetized by lack of response to toe pinch. Open the abdominal wall below the xiphoid process level using scissors. Make a transverse incision in the diaphragm, cut both sides of the ribs in the medial axillary line without any damage to the heart, and flip the anterior chest wall upwards. Advance curved forceps behind the heart, and hold the descending aorta, esophagus, and inferior vena cava. Then, excise the heart with scissors rapidly together with the adjacent vessels and tissue, such as the aorta, lungs, trachea, esophagus, adipose tissues, and thymus.

2. Wash the heart with $10 \mathrm{~mL}$ of ice-cold PBS in a petri dish, and remove the adjacent tissue.

3. Introduce the tip of the 21-gauge blunted needle connected to the perfusion circuit into the ascending aorta, and fix it with thread under a stereomicroscope.

4. Start to perfuse the heart for 10 min with Tyrode's solution aerated with $100 \% \mathrm{O}_{2}$.

5. Monitor the perfusion pressure continuously with the pressure transducer connected to the amplifier and recorder.

6. Keep the perfusion pressure between $80-100 \mathrm{mmHg}$ (usually corresponding to $2-5 \mathrm{~mL} / \mathrm{min}$ for the flow rate) during all the following steps.

7. During the initial perfusion (step 2.4.4.), insert the thin polyethylene (PE) tube (outer diameter: $0.8 \mathrm{~mm}$ ) into the SVC, and fix it with a thread. Then, place the heart into the warmed glass chamber (Figure 1b\&c). 
8. Puncture a 24-gauge indwelling needle (outer diameter: $0.7 \mathrm{~mm}$ ) into the left ventricular (LV) cavity through the ventricular apex to avoid any damage to the atrium, and remove the inner needle leaving the external cannula in the LV.

9. Introduce a 1-French size custom made electrode catheter through the PE tube in the SVC to perform electrical stimulation in the RA. If needed, advance the catheter into the right ventricle (RV) for ventricular pacing.

10. Insert a pin electrode into the ventricular apex to continuously record the bipolar electrocardiograms (ECG) between the pin electrode and cannulation needle in the ascending aorta (Figure 1c).

11. Continue monitoring the ECG and perfusion pressure throughout the entire study.

12. Turn off the light, and perform the following experiment in a dark room.

13. Go to step 2.5. for a single recording of the membrane voltage or 2.6. for a dual recording of the membrane voltage and $\mathrm{Ca}^{2+}$ transient.

5. Staining for a single recording of the membrane voltage

1. Maintain the perfusion solution heated at $37^{\circ} \mathrm{C}$ during this staining protocol for a single recording of the membrane voltage.

2. Dilute $8.3 \mu \mathrm{L}$ of the di-4-ANEPPS stock solution $(6 \mathrm{mM})$ with $10 \mathrm{~mL}$ of Tyrode's solution (the final concentration is $5 \mu \mathrm{M}$ ).

3. Infuse the total volume $(10 \mathrm{~mL})$ of the diluted di-4-ANEPPS solution into the heart via the perfusion route for $2-5 \mathrm{~min}$, followed by a washout with Tyrode's solution for 5 min.

4. Dilute $5 \mu \mathrm{L}$ of the blebbistatin stock solution $(50 \mathrm{mM})$ with $1 \mathrm{~mL}$ of Tyrode's solution (the final concentration is $250 \mu \mathrm{M}$ ).

5. Administer the total amount of the diluted blebbistatin solution via the perfusion route.

6. Skip step 2.6 and proceed to step 2.7 for washout.

6. Staining for a dual recording of the membrane voltage and $\mathrm{Ca}^{2+}$ transient

1. Keep the Tyrode's solution at room temperature.

NOTE: This initial setting of the temperature is different from that in staining for a single recording of the membrane voltage (step 2.5.).

2. Administer the blebbistatin in the same manner as in steps 2.5.4 and 2.5.5.

3. Mix $3 \mu \mathrm{L}$ of Rhod2AM stock solution (10 mM) and $30 \mu \mathrm{L}$ of polyoxyethylene-polyoxypropylene block copolymer F-127 (20\% in DMSO), then dilute the mixture with $10 \mathrm{~mL}$ of Tyrode's solution.

4. Load the total amount of Rhod2AM solution ( $3 \mu \mathrm{M})$ over $2-5$ min via the same route as the blebbistatin.

5. Dilute $7 \mu \mathrm{L}$ of $\mathrm{RH} 237$ stock solution $(10 \mathrm{mM})$ with $10 \mathrm{~mL}$ of Tyrode's solution.

6. Administer the total amount of the diluted $\mathrm{RH} 237$ solution $(7 \mu \mathrm{M})$ over $2-5$ min.

7. After completion of the above procedure, start to heat the Tyrode's solution at $37^{\circ} \mathrm{C}$.

7. Washout

1. Keep the temperature of Tyrode's solution at $37^{\circ} \mathrm{C}$ in the following experiment.

2. Perfuse the heart with Tyrode's solution for at least $5 \mathrm{~min}$ to wash out the excessive dyes.

NOTE: There is no need to increase the flow rate during this wash out step (refer to step 2.4.6.)

3. Confirm the disappearance of any motion artifact due to contractions, and the homogeneous staining in the perfused heart.

8. Sampling and data analysis

1. Put the cover glass $(25 \mathrm{~mm} \times 60 \mathrm{~mm})$ on the perfused heart carefully, to flatten the atrial surface without too much mechanical stress, and prevent motion artifact from vibration of the solution. Confirm that the atrium attaches to the cover glass appropriately.

2. Record the fluorescence by the CMOS cameras with a sampling rate up to $0.1 \mathrm{~ms}$ for di 4-ANEPPS, and $1 \mathrm{~ms}$ for the RH237 and Rhod2AM staining.

3. Analyze the obtained recordings using the analyzing software, according to the manufacturer's instructions for the actual operation of the software.

4. Create an activation map and movie after extracting the region of interest, drift removal, temporal filtering, and $3 \times 3$ binning ${ }^{5}$.

\section{Electrophysiological study}

1. Setting the pacing electrodes and stimulator

1. Confirm the contact of the tip of the custom-made pacing electrode in the RA to the tissue for stimulation (refer to step 2.4.9.).

2. Deliver all pacing stimuli from the pacing catheter connected to the programmable stimulator.

2. Determination of the pacing threshold

1. Set the pacing interval between 100 and $150 \mathrm{~ms}$ (pulse width of $0.4 \mathrm{~ms}$ ), avoiding any intrinsic rhythm outpacing the pacing rate.

2. Deliver constant pacing stimulation for at least 20 beats to obtain a stable atrial pacing.

3. Set the pacing output at $5 \mathrm{mV}$, then gradually reduce it until the delivered pacing fails to depolarize the atrium.

4. Confirm the atrial excitation by the presence of $P$ waves in the ECG, and/or an atrial excitation signal by the optical recording.

5. Determine the minimum pacing output that is able to depolarize the atrium as the pacing threshold.

6. Set the pacing output for the following studies at twice the pacing threshold.

3. Constant and burst pacing

1. Deliver constant pacing for 99 beats, with a pacing interval starting at $150 \mathrm{~ms}$, or the longest interval that avoids outpacing the intrinsic rhythm.

2. Reduce the pacing interval progressively with $5 \mathrm{~ms}$ steps, down to $40 \mathrm{~ms}$ or until reaching the interval that fails to obtain $1: 1 \mathrm{capture}$ of the atrium.

4. Single extrastimulus pacing

1. Set the pacing cycle length of the basic drive (S1) to $120 \mathrm{~ms}, 100 \mathrm{~ms}$, and $80 \mathrm{~ms}$ unless the intrinsic rhythm outpaces the basic drive or the pacing fails to obtain 1:1 atrial excitation.

2. Set the number of pacing stimuli to 10 beats for the basic drive train. 
3. Set the first extrastimulus (S2) to $-10 \mathrm{~ms}$ for the basic cycle length.

4. Deliver $\mathrm{S} 2$ after the last pacing stimulus of the basic drive.

5. Shorten the coupling interval of $\mathrm{S} 2$ progressively with $5 \mathrm{~ms}$ steps, until the $\mathrm{S} 2$ fails to depolarize the atrium.

6. Determine the effective refractory period (ERP) as the longest S2 interval that fails to depolarize the atrium.

7. Evaluate the ERP with at least 3 different basic cycle lengths to assess the rate adaptation of the ERP.

5. Double and triple extrastimuli pacing to induce atrial tachyarrhythmias

1. Reset the $\mathrm{S} 2$ interval to a point $20 \mathrm{~ms}$ outside the ERP of $\mathrm{S} 2$ if no arrhythmias are observed.

2. Add a second extrastimulus (S3), starting with the same interval as S2.

3. Decrease the coupling interval between S2 and S3 progressively with $5 \mathrm{~ms}$ steps until S3 fails to depolarize the atrium.

4. Reset the interval of $\mathrm{S} 2$ to a point $10 \mathrm{~ms}$ outside the ERP, then repeat step 3.5.3.

5. Reset the $\mathrm{S} 2$ and $\mathrm{S} 3$ intervals to points 20 ms outside each ERP.

6. Add a third extrastimulus (S4), starting with the same interval as S3.

7. Decrease the coupling interval between $\mathrm{S} 3$ and $\mathrm{S} 4$ progressively with $5 \mathrm{~ms}$ steps until $\mathrm{S} 4$ fails to depolarize the atrium.

8. Reset the $\mathrm{S} 3$ interval to a point $10 \mathrm{~ms}$ outside the ERP, then repeat step 3.5.7.

9. Reset the S2 and S3 intervals to points $10 \mathrm{~ms}$ outside the ERP, then repeat step 3.5.7.

10. Define the inducibility of atrial tachyarrhythmias by the reproducible induction using similarly programmed stimuli.

6. Evaluation of the inducibility of atrial tachyarrhythmias

1. Continuously record the ECG during all pacing protocols (step $3.3-3.5$.).

2. Perform optical recordings during and after each stimulation, to record the induction of any atrial tachycardia (AT) or repetitive atrial response (RAR).

3. Confirm the reproducibility by applying the same pacing protocol when AF or an RAR is induced.

\section{Representative Results}

The optical mapping experiments are performed using the devices as shown in Figure 1a. The schema of the optical system is illustrated in Figure 1b. The system provides a high-resolution analysis of the membrane potential and $\mathrm{Ca}^{2+}$ transient in the atrium.

As depicted in Figure 1c and $\mathbf{d}$, the isolated heart is positioned in a temperature-controlled chamber. A 1-French size electrode catheter is placed in the RA through a PE tube inserted into the SVC, and another indwelling PE tube is inserted into the LV cavity from the LV apex to avoid excessive pressure on the heart chamber. For a simultaneous ECG recording, the cathode (ECG lead [-]) is connected to the cannulation needle, and the anode (ECG lead [+]) is connected to the pin electrode inserted into the ventricular apex. The feature of this assembly is to avoid any mechanical damage to the atria.

For the evaluation of the membrane voltage, fluorescence using di-4-ANEPPS is recorded with up to a 10,000 frames/s sampling rate (Figure 2). The activation map is obtained during constant pacing delivered from the RA. The fine mapping enables the analysis of the detailed conduction pattern in small murine atria.

Figure 3 illustrates representative traces of the membrane voltages and $\mathrm{Ca}^{2+}$ transient in the LA using RH237 and Rhod2AM during constant pacing from the RA. We reduce the sampling rate of the mapping to 1000 frames/s to obtain a sufficient signal/noise ratio of the Rhod2AM signal with the present setting. However, the system provides a sufficient quality for analyzing the relationship between the action potential and $\mathrm{Ca}^{2+}$ transient.

We then perform an induction of an atrial tachyarrhythmia with programmed stimulation using mice under a pathological condition. Figure 4 exhibits an optical recording of an induced AT in a murine atrium stained with di-4-ANEPPS. The mouse undergoes a transverse aortic constriction procedure to apply a pressure overload to the atrium 10 days before the experiment ${ }^{6}$. We induce AT by triple extrastimuli pacing (120/100/100/80), and observe the propagation of the reentrant circuit. 
a.

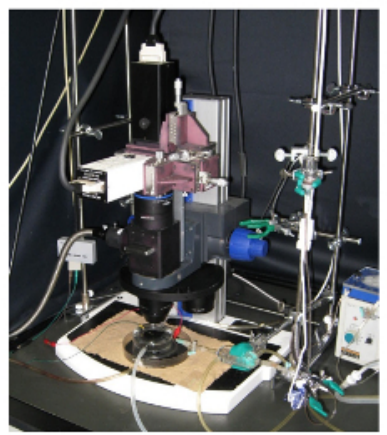

C.

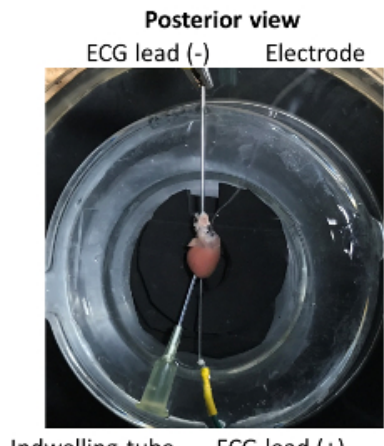

b.

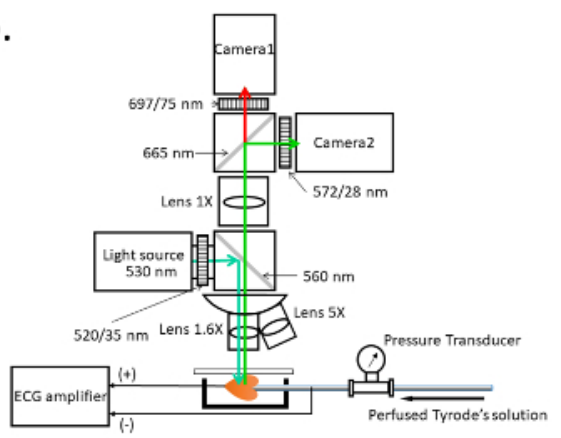

d.
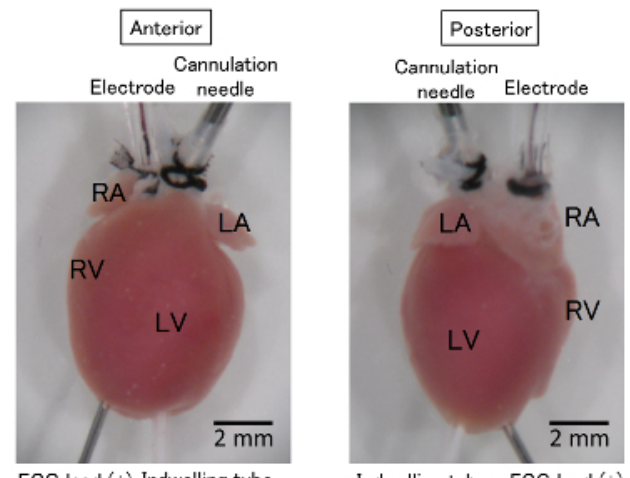

Figure 1. Optical mapping system. a. General assembly of the electrophysiology/optical mapping system. $\mathbf{b}$. Schematic of the composition of the optical mapping system: The blue arrow indicates the excitation light generated by the light source. The objective lens can be exchanged with the turret. The emitted light from the stained heart is split between camera 1 and camera 2. Camera 1 detects the long wavelength signals for the membrane voltage, and camera 2 records the wavelengths with a bandpass filter of $580 \pm 20 \mathrm{~nm}$ for the $\mathrm{Ca}^{2+}$ transient. During the procedure, the ECG and perfusion pressure are continuously recorded, and the ECG signal is also simultaneously imported into the optical map recording software. c. Preparation of the isolated hearts: The hearts are cannulated with a blunted needle via the ascending aorta for perfusion. For simultaneous ECG recordings, the cathode is connected to the cannulation needle, and the anode with the pin electrode is inserted into the ventricular apex. d. Cannulation tube and the electrodes: The heart is cannulated with a 21-gauge blunted needle for perfusion (cannulation needle). A 1-French size stimulating electrode (Electrode) is placed in the right atrium through a polyethylene tube inserted into the superior vena cava. The glass chamber is warmed at $37^{\circ} \mathrm{C}$ by heated water circulating in the cavity of the chamber. Please click here to view a larger version of this figure. 
a.

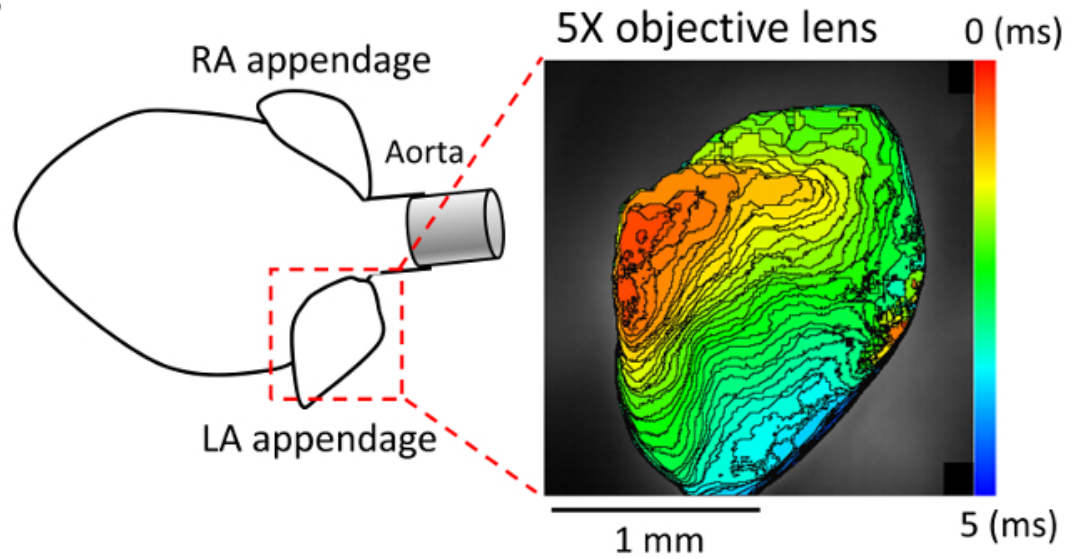

b.

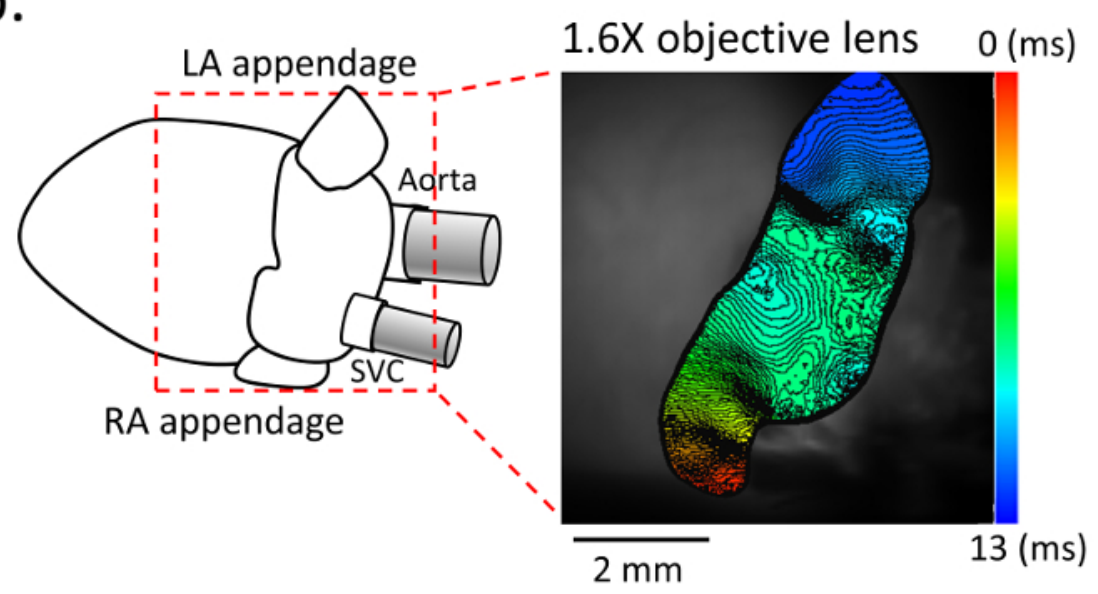

Figure 2. Activation map in the atrium. a. Representative activation maps in the anterior view with a $5 \mathrm{X}$ objective lens, and $\mathbf{b}$. in the posterior view with a 1.6X objective lens. (Left panel) Schematic of the isolated heart and atrium. (Right panel) Activation map of the left atrium obtained with di-4-ANEPPS staining at 10,000 frames/s. The recording is performed under constant pacing with the stimulating electrode placed in the right atrium. Please click here to view a larger version of this figure. 


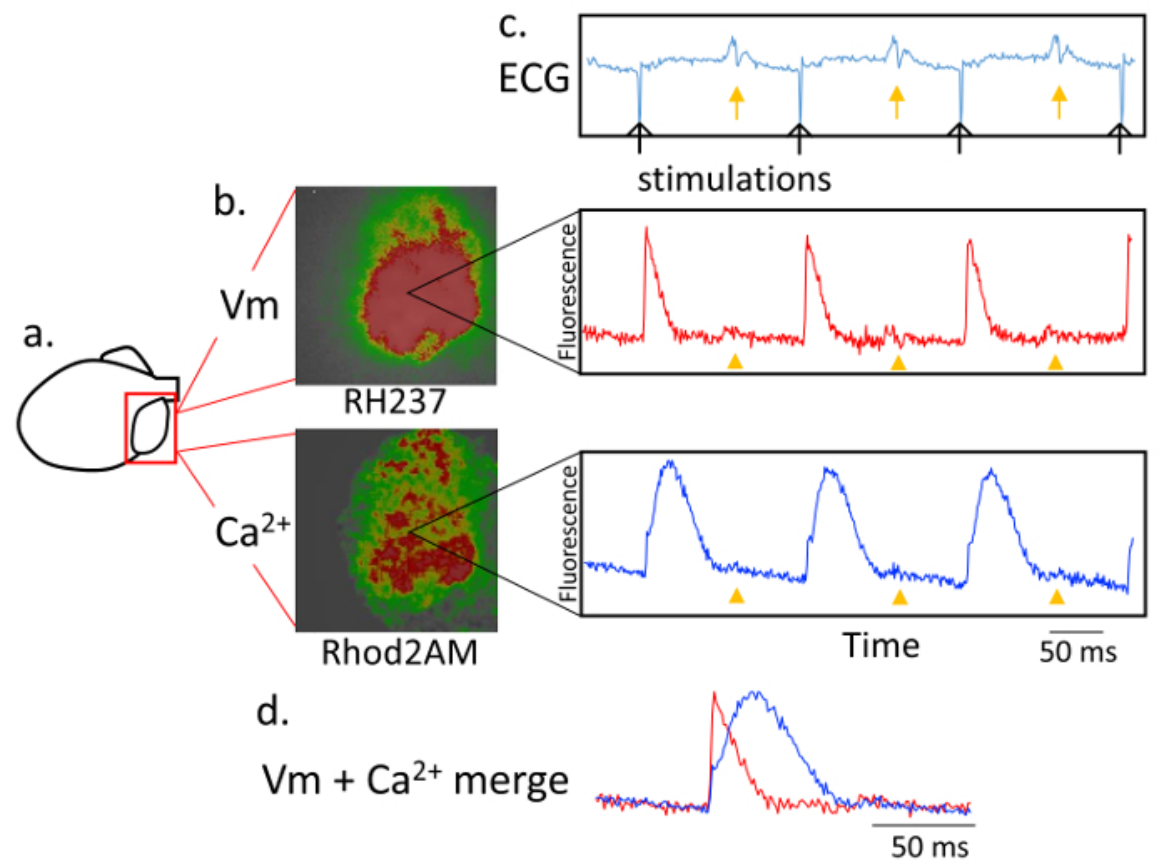

Figure 3. Dual recording of the action potential and $\mathrm{Ca}^{2+}$ transient in the left atrium. a. Schematic of the isolated heart. b. Representative image of the intensity of the fluorescence in the left atrium using RH237 for the membrane voltage $(\mathrm{Vm})$ and $\mathrm{Rhod} 2 \mathrm{AM}_{\mathrm{M}}$ for the Ca ${ }^{2+}$ transient. The recording is performed with a 5X objective lens at 1,000 frames/s. c. Simultaneous recording of the ECG (top), RH237 signal (middle), and Rhod2AM signal (bottom), during constant pacing with the stimulation electrode in the right atrium. The black arrows indicate the stimulation spikes, and yellow arrows the ventricular excitation. A light scattering effect from the ventricle also can be observed (yellow arrowheads) both in the RH237 signal and Rhod2AM signal. d. A merged RH237 and Rhod2AM trace. The action potential and transient Ca ${ }^{2+}$ changes are simultaneously recorded. Please click here to view a larger version of this figure.

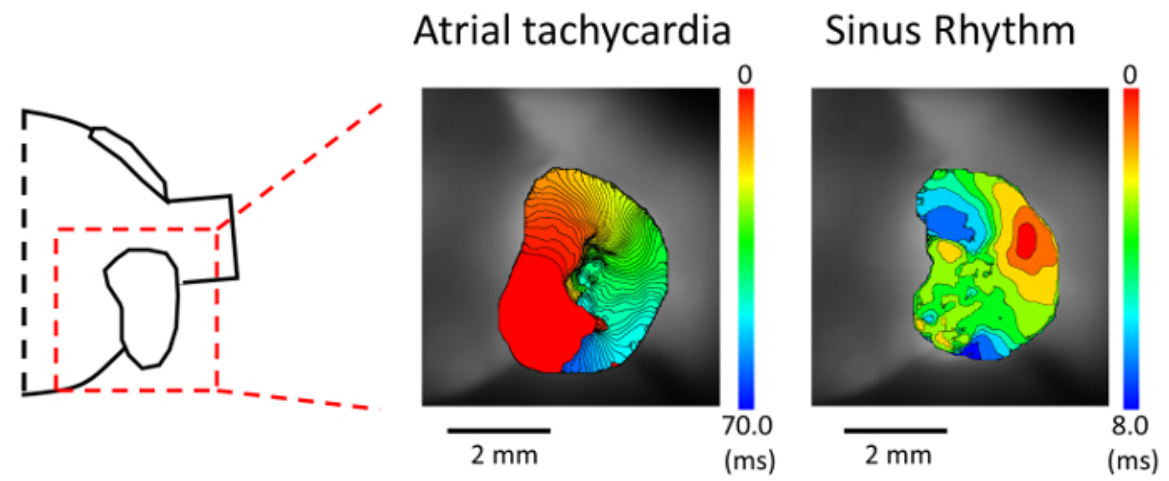

Figure 4. Activation mapping during atrial tachycardia. (Left panel) Schematic of the isolated heart. (Middle panel) Activation map during an atrial tachycardia (AT). AT was induced by triple extrastimuli pacing delivered from the right atrium. (Right panel) Activation map during sinus rhythm in the same mouse heart. Please click here to view a larger version of this figure.

Supplementary movie 1. Representative movie of the membrane potential in the atrium using staining with di-4-ANEPPS. Please click here to download this file.

Supplementary movie 2. Representative movies of the activation mapping during atrial tachycardia and sinus rhythm. Please click here to download this file.

\section{Discussion}

Optical mapping is a well-established maneuver for studying the cardiac electrophysiology ${ }^{7}$, and is a quite useful tool to assess not only ventricular arrhythmias ${ }^{8,9}$, but also atrial ones ${ }^{10,11}$. Simultaneous mapping of the transmembrane potential and $\mathrm{Ca}^{2+}$ transients is useful for understanding the underlying mechanisms of arrhythmias in relation to heart failure and other heart disease ${ }^{12,13}$. When comparing the other electrophysiological assessment methods, such as those using a single cell or cell sheet, one of the absolute superiorities of optical mapping in the perfused heart is the assessment of the conduction pattern in the intact atrium and ventricle, not only during sinus rhythm but also during induced arrhythmias ${ }^{14}$. An attempt to utilize murine hearts, especially the atrium, as a surrogate of humans has encountered difficulty mainly due 
to their small size, however, the mouse is an attractive experimental model in terms of the assessment in a genetically-engineered animal model, and this problem must be overcome. Our approach provides one direction to resolve it.

Although our optical mapping apparatus was basically similar to the conventional system for whole murine hearts ${ }^{15}$, our method has the advantage of assessing the murine atrium by making some modifications to it. First, we pursued to obtain a high spatial and temporal resolution of up to $0.1 \mathrm{~ms} / \mathrm{frame}$ and $20 \mu \mathrm{m} / \mathrm{pixel}$, and this high-resolution mapping contributed to a more precise measurement of the conduction velocity and propagation pattern in the murine atrium. Second, to avoid any unnecessary mechanical damage or stretch of the atrium, which could alter the electrophysiological properties ${ }^{16,17}$, an indwelling needle is inserted directly into the LV to reduce the intra-chamber pressure, instead of inserting it through the LA as performed in the previous study ${ }^{15}$. Furthermore, the pacing stimulus is delivered through a custom made 1French size electrode catheter placed in the RA, but not by a needle electrode, which might injure the atrium. Any pins are avoided in fixing the atrial appendage, which were used in the past study ${ }^{15}$. Third, in terms of the assessment of the underlying mechanism of the arrhythmias, a programmed stimulation protocol to induce atrial tachyarrhythmias is crucial ${ }^{18,19}$. We perform programmed stimulation identical to that in clinical electrophysiological studies, including burst pacing and up to triple extrastimuli pacing, with a modification of the pacing interval for the mouse heart. Thus, in addition to the baseline measurement parameters, the protocol could evaluate the inducibility of the AT. When needed, the inducibility of the AT is assessed with the administration of isoproterenol or other drugs. In our experience, the wild-type mice hardly show any ATs even after a full stimulation protocol. Thus, the inducibility of AT should be important information for evaluating the contribution of several pathological conditions such as genetic mutations, surgical procedures, and the administration of drugs ${ }^{11}$. Those modifications could optimize the precise electrophysiological assessment in the intact murine atrium.

This method also has some limitations. First, using a maximum spatial resolution with a $5 X$ objective lens, the field of view (FOV) is limited to a part of the atrium (i.e. only the left atrial appendage as shown in Figure 2a). For obtaining the larger FOV of the atrium, a 1.6X objective lens is sometimes preferable (Figure 2b). Second, without fixing the atrium with pins, sometimes it is difficult to measure the atrial conduction properties correctly, because the atrial surface is curved. So, we placed the cover glass on its surface to flatten it instead of fixing it by pins. This method is also beneficial for preventing motion artifact from vibrations of the solution. Third, with our method, it is quite difficult to obtain the entire FOV of it, so, to use the anterior and posterior view properly is more important in our approach than in the other approach as shown in Figure 2. The advantage of the anterior view would be the clear observation of reentry in the case of pathological conditions, especially in the appendage (Figure 4). On the other hand, the posterior view has an advantage of obtaining a good view of the atrial posterior wall, and might be a detailed recording of triggering activity from the myocardial sleeve. When it is difficult to obtain an appropriate view and to flatten its curved surface with our method, the atrium can be fixed with minimal tension by pins.

With our method, there are 3 possible problems, failure of staining, pacing, and arrhythmia induction. For failure of staining, if no or slight fluorescence is observed, you should check whether the optical mapping apparatus is correctly assembled, and whether the reagent is appropriately stored and used. The condition of the perfusion solution is also crucial, which also can affect the electrophysiological properties of the heart itself, so, the condition of solution including the $\mathrm{pH}$, temperature, and whether there was enough aeration has to be strictly monitored. It is also important to avoid any air emboli in the heart. For pacing failure, if the pacing stimuli cannot excite the atrium, researchers should check whether the wiring is correct using a circuit tester. When the pacing stimuli are correctly outputted, the problem is the contact of the electrode with the tissue. Repositioning of the electrodes can resolve the problem, and our approach using the pacing catheter makes it easy. For difficulty in arrhythmia induction, RV pacing can be used for the induction of an AT in some limited cases. Using a quadripolar electrode catheter of which the distal two electrodes and proximal electrodes can be located in the RV and RA, respectively, it is easy to change the pacing site from the $\mathrm{RA}$ to the RV. This catheter is also useful for shifting the ventricular excitation when a simultaneous ventricular activation signal masks the atrial excitation signal.

This method will contribute to assessing the genotype-phenotype interactions in the AF related genes newly found by the novel studies such as GWAS, especially for the genes with which the investigation failed to show them by other approaches. With the progress of devices and techniques, the electrophysiological properties of the pulmonary vein sleeve, which is the important source of $\mathrm{AF}^{20}$, can be assessed in the intact heart with this approach.

\section{Disclosures}

The authors have nothing to disclose.

\section{Acknowledgements}

This work is supported by the Program for Improvement of Research Environment for Young Researchers from Special Coordination Funds for Promoting Science and Technology (SCF) (to T.S.), Grants-in-Aid for Scientific Research (No. 16K09494, to T.S., No. 26293052, to T.F.) from the Ministry of Education, Culture, Sports, Science and Technology (MEXT) of Japan. We appreciate Brainvision and Mr. Kenji Tsubokura for the technical assistance, and we also appreciate Mr. John Martin for his linguistic assistance.

\section{References}

1. Gollob, M. H. et al. Somatic mutations in the Connexin 40 Gene (GJA5) in atrial fibrillation. New Engl J Med. 354, 2677-2688 (2006).

2. Gudbjartsson, D. F. et al. Variants conferring risk of atrial fibrillation on chromosome 4q25. Nature. 448, 353-357 (2007).

3. Ellinor, P. T. et al. Meta-analysis identifies six new susceptibility loci for atrial fibrillation. Nat. Genet. 44, 670-675 (2012).

4. Sinner, M. F. et al. Integrating genetic, transcriptional, and functional analyses to identify 5 novel genes for atrial fibrillation. Circulation. 130, 1225-1235 (2014).

5. Laughner, J. I., Ng, F. S., Sulkin, M. S., Arthur, R. M., \& Efimov, I. R. Processing and analysis of cardiac optical mapping data obtained with potentiometric dyes. Am J Physiol-Heart C. 303, H753-H765 (2012). 
6. Oishi, S. et al. Stretch of Atrial myocytes stimulates recruitment of macrophages via ATP released through gap-junction channels. J. Pharmacol. Sci. 120, 296-304 (2012).

7. Herron, T. J., Lee, P., \& Jalife, J. Optical imaging of voltage and calcium in cardiac cells \& tissues. Circ. Res. 110, 609-623 (2012).

8. Girouard, S. D., Pastore, J. M., Laurita, K. R., Gregory, K. W., \& Rosenbaum, D. S. Optical mapping in a new guinea pig model of ventricular tachycardia reveals mechanisms for multiple wavelengths in a single reentrant circuit. Circulation. 93, 603-613 (1996).

9. Koizumi, A. et al. Genetic defects in a His-Purkinje system transcription factor, IRX3, cause lethal cardiac arrhythmias. Eur. Heart J. 37, 1469-1475 (2016)

10. Glukhov, A. V., Uchida, K., Efimov, I. R., \& Nichols, C. G. Functional roles of $\mathrm{K}_{\text {ATP }}$ channel subunits in metabolic inhibition. J. Mol. Cell. Cardiol. 62, 90-98 (2013).

11. Takahashi, K. et al. High-fat diet increases vulnerability to atrial arrhythmia by conduction disturbance via miR-27b. J. Mol. Cell. Cardiol. 90, 38-46 (2016).

12. Choi, B.-R., \& Salama, G. Simultaneous maps of optical action potentials and calcium transients in guinea-pig hearts: mechanisms underlying concordant alternans. J. Physiol. 529, 171-188 (2000).

13. Hwang, G.-S. et al. Intracellular calcium and vulnerability to fibrillation and defibrillation in Langendorff-perfused rabbit ventricles. Circulation. 114, 2595-2603 (2006).

14. Kwaku, K. F., \& Dillon, S. M. Shock-induced depolarization of refractory myocardium prevents wave-front propagation in defibrillation. Circ. Res. 79, 957-973 (1996).

15. Lang, D., Sulkin, M., Lou, Q., \& Efimov, I. R. Optical mapping of action potentials and calcium transients in the mouse heart. J. Vis. Exp. (55), e3275 (2011).

16. Eijsbouts, S. C. M., Majidi, M., Zandvoort, M. v., \& Allessie, M. A. Effects of acute atrial dilation on heterogeneity in conduction in the isolated rabbit heart. J. Cardiovasc. Electr. 14, 269-278 (2003).

17. Ravelli, F., \& Allessie, M. Effects of Atrial dilatation on refractory period and vulnerability to atrial fibrillation in the isolated Langendorffperfused rabbit heart. Circulation. 96, 1686-1695 (1997).

18. Sasano, T., McDonald, A. D., Kikuchi, K., \& Donahue, J. K. Molecular ablation of ventricular tachycardia after myocardial infarction. Nat. Med. 12, 1256-1258 (2006)

19. Wakimoto, H. et al. Induction of atrial tachycardia and fibrillation in the mouse heart. Cardiovasc. Res. 50, 463-473 (2001).

20. Haissaguerre, M. et al. Spontaneous Initiation of Atrial Fibrillation by Ectopic Beats Originating in the Pulmonary Veins. New Engl J Med. 339, 659-666 (1998). 